\title{
Cultura literaria transfronteriza hispánica en Portugal: vestigios documentales actuales del Siglo de Oro en el territorio oriental de Trás-os-Montes

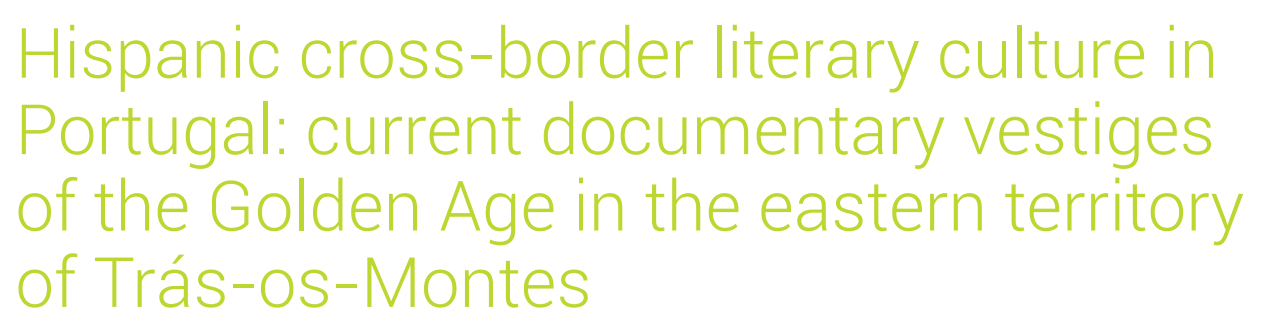

\section{Alexia Dotras Bravo}

Instituto Politécnico de Bragança

Centro de Literatura Portuguesa

alexia@ipb.pt

[Hipogrifo, (issn: 2328-1308), 7.1, 2019, pp. 597-613]

Recibido: 04-02-2019 / Aceptado: 04-03-2019

DOI: http://dx.doi.org/10.13035/H.2019.07.01.45

Resumen. El presente trabajo pretende dar cuenta de la existencia de los fondos antiguos conservados que existen en el Arquivo Distrital de Bragança, de cerca de cinco mil ejemplares, que abarcan del siglo XVI a inicios del siglo XX. En primer lugar, se analizará la posición estratégica de frontera de Bragança entre los siglos XVI y XVII, período clave, no solo para el Siglo de Oro, sino también para la relación política e histórica de Portugal y España (1580-1640/1668). En segundo lugar, se divulgará y estudiará el conjunto de los volúmenes en español del fondo, además de la biblioteca personal de Francisco Manuel Alves, abad de Baçal, sita en el centro de secundaria Emídio Garcia. Por último, con el ejemplo constante del abad, sus lecturas y referencias librescas hispánicas del Siglo de Oro, se estudiará y reflexionará sobre la verdadera cultura literaria española en Portugal.

Palabras clave. Bragança; archivos con fondos antiguos; Francisco Manuel Alves; cultura literaria transfronteriza; literatura española en Portugal. 
Abstract. The present work pretends to give account of the existence of the conserved old funds that exist in the District Archive of Bragança, of near five thousand units, that goes from the 16th century at the beginning of the 20th century. Firstly, Bragança's strategic border position will be analyzed between the 16th and 17th centuries, a key period not only for the Golden Age, but also for the political and historical relationship between Portugal and Spain (1580-1640 / 1668). Secondly, the set of volumes in spanish of the fund will be communicated and studied, as well as the personal library of Francisco Manuel Alves, abbot of Baçal, located in the secondary school Emídio Garcia. Finally, with the constant example of the abbot, his readings and hispanic bookish references of the Golden Age, the true spanish literary culture in Portugal will be studied and reflected.

Keywords. Bragança; Archives with old funds; Francisco Manuel Alves; Cross-border Literary culture; Spanish literature in Portugal.

\section{BRAGANÇA, CIUDAD TRANSFRONTERIZA EN LOS SIGLOS XVI Y XVII}

De entre todos los enclaves fronterizos hispano-portugueses en más de 1200 kilómetros de frontera, Bragança es una región, la más alejada de Lisboa, de peculiaridades orográficas, físicas, gastronómicas, lingüísticas y folclóricas que generan dos visiones contrapuestas entre ellas. Para el portugués del litoral o de los centros urbanos más populosos, se trata de una ciudad y un distrito eminentemente rurales, poco evolucionada tecnológica, económica y socialmente, y excesivamente pequeña. Es decir, todas aquellas características que se le atribuían a Portugal hace años, estigmas de los que se ha venido librando en las dos últimas décadas, y que ya no quiere ostentar hoy, siendo como es uno de los destinos turísticos mundiales más atractivos. Sin embargo, para el brigantino esta es una percepción errada de todo punto.

Para el bragançano, entonces, es una ciudad con muchas posibilidades, con mucho más que dar de lo que consideran fuera de la región. Como reza la publicidad turística municipal, es un «territorio por conquistar» ${ }^{7}$. Lema este que remite a varios rasgos clave en la identidad brigantina: por un lado, al ser una de las grandes desconocidas para el resto del país, todavía está por "conquistar»; por otro, se enorgullece de haber ofrecido gran resistencia a todas las guerras fronterizas en los diferentes momentos históricos donde hubo de resistir desde su castillo y desde los parajes agrestes de sus montañas.

Algunas de las visibilidades de la ciudad se dan en ámbitos de meridiana contemporaneidad, para demostrar que es una ciudad moderna, o incluso más allá de moderna. Así, surgió en 2015 como una de las cuatro smartcities portuguesas, para sorpresa del resto del país, incluso de aquellos de origen transmontano afincados en otras regiones más desarrolladas:

1. Un compendio completo de la autopercepción estratégica actual y de la proyección consciente que se quiere dar a la ciudad se encuentra en el suplemento de la revista Villas\&Golfe, 90, novembro 2016. 
Porquê surpreendidos? Primeiro, porque há ainda um estigma e uma aversão elitista nas grandes cidades do litoral para as pequenas cidades periféricas do interior. Há uma diáspora inteira (e sublinho diáspora) que ainda pensa que as suas terras natais se mantêm na mesma, como quando as deixaram rumo às novas paragens em busca de vidas melhores².

La transformación de Bragança, para salir de un letargo propio de ciudad de interior, es evidente y así lo atestigua la bibliografía local. Contrastes e transformações da cidade de Bragança 1974-2004³ es una de esas obras que ambicionan dar fe de una situación de cambio total para la capital de distrito que quiere ser hoy: con el mejor centro de enseñanza superior politécnica del país (IPB), un polo industrial de automoción de referencia ibérica (Faurecia y las empresas proveedoras del sector del automóvil subsidiarias a esta), una incubadora de empresas emprendedoras (Brigantia-Ecopark), la referencia internacional en investigación agroalimenticia (CIMO) que, de alguna forma indeliberada, relegan a un segundo plano discreto las inversiones culturales. No obstante, para hacer honor a la verdad, el pasado histórico y cultural forma parte del orgullo de bragançanos, sobre todo en el ámbito folclórico y tradicional, algo alejado de la sofisticación de la cultura urbanita. Con todo, instituciones públicas y privadas se esfuerzan en crear una vida cultural inédita en una pequeña ciudad de interior, ejemplificados gráficamente en el hecho de poseer siete museos o centros de arte y estar en proyecto el ambicioso Museu da Língua Portuguesa. El problema reside en la adhesión de la ciudadanía a los proyectos culturales no folclóricos, más inclinados a apoyar causas que manifiesten un evidente progreso. El brigantino prefiere ser distinguido en el contexto nacional por una vía tecnológica moderna que por elementos patrimoniales abstractos o de minorías, actitud propia del miedo a caer en el olvido social y la necesidad del reconocimiento público.

Este aspecto cultural es el que me interesa, específicamente en su relación con la historia y su vinculación de amor-odio con la frontera. Desgraciadamente, se trata de un compromiso desde las fuerzas y poderes públicos, que la sociedad en su mayoría no secunda. Bragança se presenta a los ojos de la opinión pública como una ciudad con siglos de historia, marcada por una peculiar orografía, tradiciones y formas lingüísticas. Así se colige de la documentación local, centrada en el antiguo fuero que le confiere rango de ciudad ${ }^{4}$ o la protohistoria brigantina, bien documentada y siempre realista ante la interioridad que la sume en claras regresiones sociales, económicas y culturales ${ }^{5}$. Sentimientos encontrados chocan en estas obras de claro sabor localista, subjetivo y embellecedor. Al referirse a su historia, la contradicción evidente se instala para puntualizar esa inevitable mención de la frontera, pero que bien saben resumir los que viven los confines desde dentro:

\footnotetext{
2. Pereira, 2016, p. 96.

3. Fernandes, 2004

4. Nunes, 2009.

5. Rodrigues Monteiro, 1988
} 
Em determinados períodos da sua história, Bragança beneficiou da sua posição geo-estratégica e foi, durante o período de consolidação de fronteiras, como praça militar fronteiriça, várias vezes saqueadas e outras tantas reconstruída. A frontera isolou-a, colocando-a geográficamente numa posição periférica face aos principais mercados e centros de decisão política e económica nacionais. Com a adesão à CEE no ano de 1986, a extinção de fronteiras e a adopção de políticas sociais e económicas de âmbito europeu, Bragança readquiriu uma nova centralidade no contexto ibérico ${ }^{6}$.

La frontera aparece, por fin, en toda su proyección. Una comunicación secular basada en el conflicto (en los lazos de sangre, en las herencias, en los amores) de la tierra ${ }^{7}$, pero sobre todo en los trances bélicos habituales desde la Edad Media. Conflictos que no impiden que la frontera en sí sea un espacio de sobrada acción común.

Justamente en esta frontera se libran algunas batallas con España que son especialmente intensas en esta zona limítrofe a lo largo de la historia, a pesar de que históricamente se atribuye esta lucha más hacia el sur. Así lo atestigua Francisco Manuel Alves, conocido como abad de Baçal, desde los inicios de la creación de los reinos hasta la revolución de 1820. Dedica especial esfuerzo a las guerras tras la Restauración monárquica portuguesa (1640-1668), época que nos interesa especialmente y que son un ejemplo vívido del concepto bélico de frontera:

\footnotetext{
O texto que daí resultou continua a ser imprescindível para o conhecimento das acções militares desenvolvidas num lado e noutro da fronteira, muitas vezes conduzidas sem ordem nem regra; mas houve-as programadas e apoiadas em linhas estratégicas bem definidas. Contrariamente ao que às vezes se pensa, não foi apenas na fronteira do Alentejo que a sorte das armas portuguesas se definiu, neste longo pleito. As fronteiras do Minho e Trás-os-Montes conheceram acções de alguma envergadura ${ }^{8}$.
}

A esas luchas de frontera en las guerras de 1640, tras la vuelta de Portugal a la corona lusa, llamadas por él de Aclamação, dedica el abad todo un capítulo del primer tomo de sus Memórias y bastante espacio del octavo, de claro sabor español. Hay una constante en todo su trabajo a lo largo de siglos de historia, que se basa en la primacía de la frontera de Miranda sobre Bragança y, por tanto, de la preeminencia social, política y económica, que ya hemos señalado:

el abad sí transcribe bastantes cartas y órdenes reales dando privilegios a habitantes del distrito, especialmente de Miranda, para poder comerciar libremente con las tierras cercanas (Zamora, Aliste, Benavente, Sayago, Alcañices y otros) (...), dando más libertad a la hora de comprar y vender animales y trasladar dinero a un lado y otro de la frontera. Tales cartas datan de 1521 y 1524, sin incluir tierras de Sanabria, probablemente por la dificultad de atravesar las montañas, tal y como hoy en día ${ }^{9}$

6. Nunes, 2009, p. 8

7. Sousa, 2013, pp. 785-798

8. Ribeiro da Silva, 2000, pp. CLXI-CLXII.

9. Dotras Bravo, 2016a, p. 121 
En todo el relato a lo largo de sus Memórias aporta bastantes documentos de pedido de dinero al cabido de Miranda para la defensa de las fronteras, no solo transmontanas, sino en repetidas ocasiones para el Alentejo. Esto refuerza la idea de la supremacía estratégica y financiera del noreste luso, más en Miranda que en Bragança, y los requerimientos por parte de otros puntos geográficos, que no se refleja en la historiografía portuguesa sobre la frontera. Es más, las relaciones fronterizas nordestinas en muchos otros aspectos que no sean los bélicos «constituem uma página ignorada do relacionamento de portugueses e espanhóis nesta parte da frontera» ${ }^{10}$.

Sin embargo, las batallas ofrecen también panoramas desoladores. Aldeas, campos, villas quemados o saqueados de ambos lados. Destaca que sea la parte gallega se muestre muy beligerante, frontera a Vinhais, Moimenta y hasta Chaves. ¿Será esta una de las causas históricas por la que Bragança establece lazos de amistad y cooperación con tierras zamoranas, más que con Galicia? La respuesta sería que no, ya que «o mesmo sistema de guerrear continuava nas fronteiras de Miranda do Douro, que tinha pelo lado contrário o marquês de Alcanices e o conde de Alva de Liste» ${ }^{11}$. Con todo, la descripción es de una auténtica guerra de batallas locales; sirva esta de muestra de mucha documentación conservada de los enfrentamientos bélicos en la frontera, trascritos y estudiados por el abad:

No princípio, esta guerra de incursões era, especialmente na nossa província, uma perfeita razia: incêndios, roubos, morticínios sem consideração alguma, de maneira que tão desastrosa era para um lado como para o outro, sem dar vantagens decisivas, porque se pelejava sem forma, sem arte e sem disciplina, como diz o autor que nos serve de guia nestas campanhas.

Os nossos entravam, roubavam, destruíam, incendiavam, matavam; para logo os galegos responderem no mesmo diapasão ${ }^{12}$.

\section{LOS FONDOS ANTIGUOS EN BRAGANÇA: DEL ARQUIVO DISTRITAL DE BRAGANÇA A LA BIBLIOTECA DEL ABAD DE BAÇAL}

Hace tres años descubrimos, o para ser más exacta, conocimos la existencia de un fondo bibliográfico desconocido para una aplastante mayoría de la ciudadanía, en el Arquivo Distrital de Bragança (ADB) que abarca del siglo XVI al siglo XX, compuesto de unos 5.000 volúmenes, cuyo registro archivístico estaba sin realizar. Por eso no tenían los números habituales de depósito, estantería y posición en la estantería, tal y como es habitual en bibliotecas con ejemplares antiguos, ni mucho menos la signatura indicada en un tejuelo. Tal acometido fue realizado tras nuestro interés por el depósito; interés y trabajo esforzado por parte de Zaida Vila Carneiro y yo misma. 
Una primera aproximación nos llevó a revisar los trabajos ya realizados por un grupo de investigadoras de la Universidade de Coimbra. Es de justicia señalar, entonces, que hace años, en los noventa, esa expedición bibliotecaria estudió e intentó catalogar algo menos de la mitad de este fondo, de la estantería primera a la 170; sin embargo, no es posible acceder a la base de datos confeccionada debido a que faltan algunos pasos fundamentales administrativos. Lo que sí podemos afirmar es que la documentación derivada de tal catalogación, en concreto las fotocopias de las portadas, resulta especialmente confusa, ya que estas están repetidas y poco organizadas. A causa de esta ausencia de sistematización, nuestra idea inicial de emprender una búsqueda exhaustiva de los libros escritos en español en la mitad restante del ADB (de la 171 a la 334) hubo de modificarse. Por lo tanto, decidimos volver a examinar todos los libros del depósito $\mathrm{A}$ y respetar el orden en que estaban colocados en la estantería, aunque este no tuviese en cuenta la cronología o la pertenencia a una colección, que se podía encontrar dispersa por el archivo, como así verificamos en algunos casos. Con todo, la investigación se dividió en dos fases y comenzó en 2016 por la estantería 171. Ya a partir de 2017 se comenzó por el principio, atendiendo a la reduplicación de portadas fotocopiadas, que dificultaba el trabajo de búsqueda. Al no tener estipulada una signatura o la anotación de una referencia mínima, el registro a lápiz en el canto superior de la primera página, se hizo a partir del número de estante y número de la posición dentro del mismo por uno de los técnicos del archivo al mismo tiempo que nosotras documentábamos y analizábamos cada ejemplar.

El primer objetivo se destinó a la catalogación y estudio de parte de esas obras, las escritas en lengua española. El amplio panorama de volúmenes, preferentemente píos, presenta cierta consistencia en el tipo de literatura leída desde el siglo $\mathrm{XVI}$, esto es, de formación religiosa y moral, especialmente la instrucción de sacerdotes y la abundancia de obras hagiográfıcas. Podemos afirmar de forma definitiva y sin ningún género de duda, una vez que ya hemos examinado todo el fondo, que las obras españolas originales ascienden a la cantidad de 498, sin contar las españolas traducidas al portugués $u$ otras lenguas, ni las latinas publicadas en ciudades españolas, ya que era este idioma el de la ciencia y la divulgación como lengua franca. De todas estas obras extraemos un total de 317 títulos, ya que muchos son los diversos volúmenes de un mismo título (Gráfico 1). Algunos autores y obras aparecen repetidas veces, por lo que podemos deducir que son manuales de estudio en el Seminario de Bragança o títulos populares entre los lectores avisados de la ciudad o de acceso fácil. Tal es el caso, llamativo, de la frecuente aparición de las Siete partidas de Alfonso X el Sabio en sus ediciones de 1555, 1565 y 1587 o la cantidad de ejemplares de Theatro crítico universal de Benito Jerónimo Feijoo, complementados por las defensas que partían de Martín Sarmiento, así como los ataques de Salvador José Mañer. El interés por este estudioso no se queda por aquí, ya que se conservan muchos ejemplares de la revista Mercurio histórico y político, revista española de la que era fundador, y una de las que más tiempo existió en la corte madrileña en el siglo XVIII. 


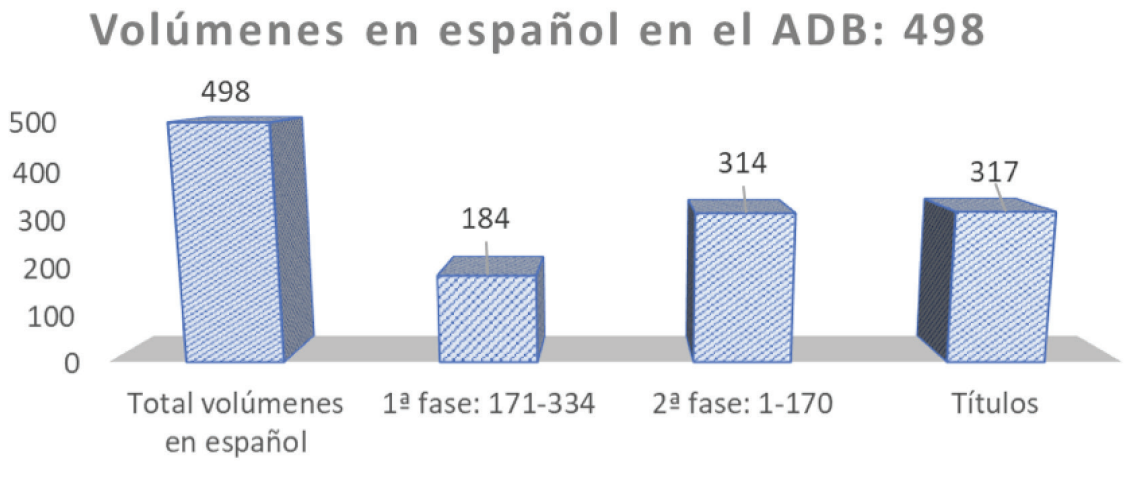

Grátıco 1: Relación del total de obras (volúmenes y títulos) en español y la cantidad de obras encontradas en cada fase de la investigación

Las materias a las que se refieren las obras en español del ADB, tras una primera aproximación, no exenta de polémica -resulta difícil separar lo moral de lo teológico, o decidir qué es literario y que es religioso en un texto místico, por ejemplo- decidimos que se daban ciertos ejes temáticos recurrentes. Los más habituales son religioso, con su vertiente moral, y político, histórico, jurídico y literario, en este orden. De hecho, la conjunción religioso-moral-político presenta unos lindes bien difusos, ya que destacan textos que buscan la formación ética y moral del dirigente político, dentro de un contexto de absoluta primacía de la doctrina católica en los asuntos de estado. En esta labor de vaciado destacan también otras temáticas agrupadas, como el cultivo y estudio de la seda, tanto en trabajos técnicos y científicos del siglo XX, como algunos históricos a lo largo de los siglos. Además, tenemos que tener en cuenta la no desdeñable atención ofrecida a China en obras antiguas, ya que aparecen varios ejemplares que relatan la historia de esta nación. Probablemente se deba a este interés por la seda, industria de gran valor histórico en Bragança, cuyo centro museológico «Casa da seda», perteneciente al «Centro Ciência Viva», lo confirma ${ }^{13}$. La historia sericultora de Bragança existe desde los comienzos del asentamiento urbano en la zona:

A indústria das sedas em Portugal, durante a Idade Média, embora sem a importância assumida pelas indústrias do linho e lanifícios, teve em Trás-os-Montes e muito particularmente em Bragança, o seu principal centro de produção. Com efeito, a mais antiga referência à criação do bicho da seda diz respeito a Trás-os-Montes, no século XIII, e as primeiras indicações quanto ao fabrico da seda, que datam do século $X V$, referem-se a Bragança ${ }^{14}$.

Por otro lado, la temática religiosa, la más frecuente (Gráfico 2), se puede desglosar en varios asuntos interligados, pero diferentes. En un primer contacto, resaltan la gran cantidad de libros hagiográficos, probablemente relacionados con

13. En su página web (<http://www.braganca.cienciaviva.pt/seda/>) inicia la explicación refiriéndose a China y a su relación milenaria con la seda (consultado el 21 de marzo de 2019).

14. Sousa, 2006, pp. 27. 
una idea clara de formación e instrucción cristianas, la segunda gran línea clara. La voluntad preceptiva domina en todo el fondo, aunando moral y religión, además de política y religión, siguiendo estelas de formación de políticos como Castiglione, Maquiavelo u otros. En menor medida, pero con una presencia notable, sobresalen obras de filosofía católica y contenido doctrinal, así como de derecho canónico.

\section{Fondos españoles en el ADB: 498}

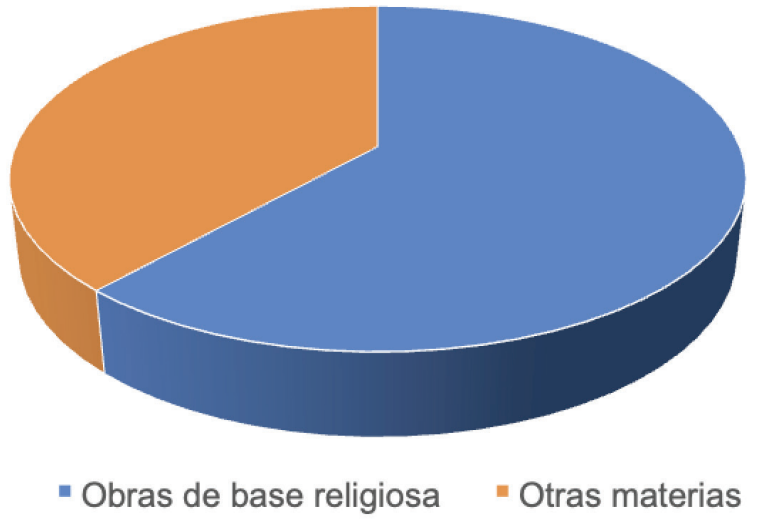

Gráfico 2: Relación temática de los fondos antiguos del ADB. Religión vs. Otras materias

Lo que inicialmente se convirtió en un atractivo añadido a la actividad investigadora habitual se fue convirtiendo en una posibilidad más estable de ofrecer temas de investigación para pequeños y no tan pequeños proyectos. A medida que íbamos anotando las obras en un documento ofrecido por la dirección del Archivo, con los elementos que aquel documento seleccionaba, pudimos observar que existían algunas similitudes en temática y en ciertas premisas comunes: en primer lugar, se trataba en un porcentaje muy alto de textos religiosos, en segundo lugar, destacaban algunos textos por repetición de temática sorprendente o singular, como ya se ha referido, y en tercer lugar, había mucha más abundancia de textos del siglo XVII y XVIII que de los otros siglos.

En los dos siglos XVI y XVII, de 1500 a 1700, podemos contabilizar como seguras 150 obras, 17 en el siglo XVI y 133 en el XVII. A estas tendremos que añadirle un número significativo de obras en mal estado o con faltas de datos. Siguiendo la división temática que hemos establecido, y pudiendo poner reparos a las adjudicaciones de obras en uno u otro eje, la distribución corrobora lo que la visión panorámica ofrecía: abundancia aplastante de textos de moral y religión de autores no literarios e interesante selección literaria, jurídica, histórica y política, en la que destacan algunas obras. En primer lugar, la ya citada frecuencia de las Siete Partidas de Alfonso X, obras sobre el contrabando y China, que indican claramente que la posición estratégica de frontera influye en la educación literaria de sus hombres doctos, interés por la iglesia protestante y la repetición de un mismo autor literario, 
Antonio de Guevara, en los dos siglos, del que se conservan tres obras. Desde una perspectiva estadística la distribución es como sigue:

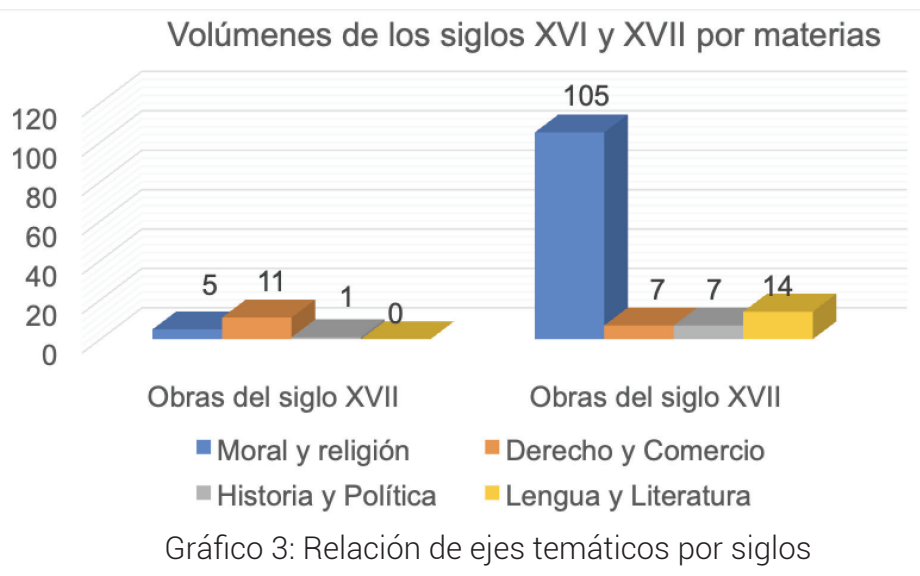

De todas formas, como ya hemos adelantado, este es un número completamente provisional. Sin fecha en portada, solo extraída de los preliminares (la tasa, la fe de erratas, la censura) hay 43 ejemplares, la mayor parte del XVII, en mal estado y sin datos que figuren por faltar la portada y hojas iniciales. De estos 43 , tres son del siglo XVIII y uno probablemente de finales del XIX o principios del XX, ya que pertenece a la editorial Calleja, fundada en 1885. Los otros 39, salvo alguna excepción, pertenecen a los siglos XVI y XVII. El análisis profundizado de estas obras excede estas líneas, pero podemos apuntar datos interesantísimos. De los más de treinta libros del Siglo de Oro podemos subrayar una mayor presencia literaria de la que arrojan los primeros datos fehacientes, gracias a la existencia de más obras de Antonio de Guevara, un volumen de comedias y traducciones de Tito Livio y Virgilio. Son volúmenes en peor estado, quizás por el uso o por no ser de la temática favorita de sus propietarios. En posteriores investigaciones estaremos en condiciones de ofrecer el catálogo con el estudio de las obras más destacadas en los ámbitos literario, histórico y cultural.

En definitiva, surgieron unos primeros frutos científicos ya en 2016, sin otra finalidad que traer a la luz unas primeras investigaciones acotadas a una obra, una época o una recepción determinada. Lo que resultó evidente es que fue necesario vincular al más importante erudito de la región, Francisco Manuel Alves, ya mencionado, con este fondo compuesto por ejemplares de la biblioteca de la Mitra, de la antigua Junta General y del Seminario de Bragança, además de las bibliotecas personales de algunos obispos célebres. En esos primeros trabajos ${ }^{15}$ los datos sobre el archivo no estaban completos, e incluso aparecen diseminados en propuestas más globales sobre Portugal y la literatura clásica española ${ }^{16}$, ya que siempre ha sido un work in progress, hasta este momento en que podemos dar informaciones fidedignas y definitivas. Sin embargo, nos sirvió para contextualizar mejor una de 
las figuras sociológicas más interesantes del pasado siglo, el erudito local, Francisco Manuel Alves en el caso de Bragança ${ }^{17}$. Tenemos que agradecer la posibilidad de llevar a cabo este trabajo de campo en el contexto del libro antiguo al convenio de investigación firmado entre la Direção Geral do Livro, dos Arquivos e das Bibliotecas portuguesas y la Escola Superior de Educação de Bragança, por un lado, y por otro, a la dirección de la Escola Secundária Emídio Garcia, heredera del Liceu, y que conserva la biblioteca personal del abad de Baçal, la cual rondará los 2500 libros, y que nos permitió, a través de un convenio de formación, colocar a una alumna en prácticas, Joana Amaro, que realizó la búsqueda y catalogación de los volúmenes en español (y también inglés) de la biblioteca, legada al Liceu de la ciudad. Este trabajo de fin de licenciatura permite contabilizar los textos del ámbito hispánico, 188 de 194 (del siglo XVII al XX), lo que evidencia una mayoría aplastante, además de resultar notable que sean 30 en gallego o sobre cultura gallega. Nos encontramos pues, con un total de 656 obras en español entre dos bibliotecas brigantinas de fondo antiguo.

De forma más concreta, los asuntos ya investigados, se centran en el Barroco tardío y las representaciones teatrales musicales en el siglo XVIII en la corte de Fernando $\mathrm{VI}^{18}$ y sobre su relación con el mundo jesuítico y tridentino ${ }^{19}$. Sin embargo, gracias al análisis interrelacionado de ambas bibliotecas, el acceso a los documentos de la época para deshacer mitos improbables -e improbados ${ }^{20}$ - y el descubrimiento de Alves como cervantista ${ }^{21}$, considero que son los logros más interesantes para el estudio de la cultura literaria siglodorista de esta zona fronteriza.

\section{UN EJEMPLO DE CULTURA LITERARIA CLÁSICA TRANSFONTERIZA: EL SIGLO DE ORO Y FRANCISCO MANUEL ALVES}

En primer lugar, hemos descubierto nuevos datos de Francisco Manuel Alves como cervantista, que se habían escapado a los organizadores de la edición moderna. Hemos analizado y agrupado las quince veces que el abad menciona a Cervantes, gracias a los índices onomásticos de las Memórias, que se erigen al lado de la improbada afirmación de que Cervantes ha estudiado en Bragança, asunto que ya hemos esclarecido en otras páginas ${ }^{22}$. Esto quiere decir que la importancia de Cervantes en Bragança no pasa por su existencia en la ciudad, sino por la recepción que ilustres eruditos hicieron de su obra, sobre todo en aspectos folclóricos, lingüísticos y etnográficos ${ }^{23}$. Sin embargo, renovadas lecturas de los once tomos, permiten descubrir referencias no aparecidas en el último volumen de índices.

17. Dotras Bravo, 2018.

18. Dotras Bravo, 2016b.

19. Dotras Bravo, en prensa.

20. Dotras Bravo, 2016a.

21. Dotras Bravo, 2018.

22. Dotras Bravo, 2016a.

23. A este respecto, se pueden consultar otras bibliotecas cervantinas, cuya lectura destaca por lecturas filológicas y etnográficas, tal y como se puede apreciar en la de Carolina Michaëlis de Vasconcelos, conservada en el Centro de Literatura Portuguesa de la Universidade de Coimbra (Dotras Bravo, 2012). 
Por ello, debemos volver a Alves como cervantista, y más concretamente, al Alves etnógrafo y lingüista.

En el tomo correspondiente a Arqueologia, Etnografia e Arte (tomo X) encontramos ahora una detallada lista del uso del término majada por Cervantes -equivalente al portugués malhada- en su relación con los pastores, su lugar de guardar el ganado o su simple reunión en un local determinado, para explicar el topónimo bragançano Malhadas. Da hasta cinco ejemplos del término, con sus correspondientes citas del Quijote de la primera parte. Se sumaría, por tanto, a las autoridades lingüístico-etnográficas de picote, lampaça, silo y senda ${ }^{24}$.

El segundo tomo del que se nos habían escapado ciertas reflexiones es el XI, Arqueologia e Etnografia, que presenta una orientación folclórica evidente. Nuestra sorpresa no es poca al ver que la referencia huidiza es de tipo escatológica, entre el capítulo XLVIII y el XLIX de la primera parte, en aquel momento en que don Quijote va enjaulado y encantado. Sancho, para probar a su amo que no es verdadero encantamiento, argumenta que la necesidad de defecar del caballero demuestra su estado normal. Las referencias al mal olor («fatigalles el olfato» ${ }^{25}$ ) y al defecarse encima («Sácame deste peligro, que no anda todo limpio!» ${ }^{26}$, «si no le dejaban salir, no iría tan limpia aquella prisión como requiría la decencia» ${ }^{27}$ ), además de ser usadas para el abad como ejemplo de naturalidad en lo que Alves denomina «alta literatura» ${ }^{28}$, nos vuelven a remitir a la lectura paródica y cómica de la obra, primera de sus intenciones según palabras del propio Cervantes.

¿Qué sucede en el resto de autores del Siglo de Oro? ¿La misma importancia como referencia etnográfica y filológica o, por el contrario, se trata de alusiones literarias, comentarios exegéticos o evidente erudición?

De los tres nombres que faltarían en la cima del Siglo de Oro (Quevedo, Góngora y Lope), el enfoque ofrecido por Francisco Manuel Alves es bastante similar al de Cervantes. No se encuentran registros de Calderón ni de Tirso ni de otros autores siglodoristas. Vamos a tratar de esta cuestión en detalle, como ejemplo de creación de un referente cultural, tanto ajeno como propio. Es decir, las menciones de los tales escritores sirven de autoridad para refrendar usos y costumbres portugueses, borrando cualquier frontera cultural entre ambos países. Si Cervantes lo ha dicho, no interesa si lo ha hecho como portugués o como español, es importante porque lo ha hecho desde un sustrato común - probablemente ibérico- anterior a nacionalidades y fronteras.

A Lope no lo menciona, sí en cambio a Quevedo, unas tres veces, mientras que a Góngora solo una. La forma de abordar a los escritores se asemeja a la que realiza con Cervantes. De memoria, como una lectura que se hace cercana y para objetivos etnográficos o lingüísticos. No obstante, la diferencia es sustancial.

24. Dotras Bravo, 2018, pp. 247-249.

25. Cervantes Saavedra, Don Quijote de la Mancha, p. 614.

26. Cervantes Saavedra, Don Quijote de la Mancha, p. 612.

27. Cervantes Saavedra, Don Quijote de la Mancha, p. 613.

28. Alves, 2000, tomo XI, p. 254.

HIPOGRIFO, 7.1, 2019 (pp. 597-613) 
Góngora aparece en el tomo VIII, uno de los más españoles en cuanto a su asunto, debido a que analiza la documentación encontrada en el archivo de Simancas sobre Bragança y retoma la Guerra de 1640 que había abordado al detalle en el primer volumen, habida tras la Restauración monárquica portuguesa y el fin de la monarquía dual. En el mismo se encuentra una de las referencias a Quevedo.

Tras una larga arenga de evidente lusofilia sobre el polémico momento del reconocimiento del rey español por parte de los portugueses de Moncorvo e de Freixo de Espada à Cinta en 1580, gracias al acta conservada en el archivo de Simancas, realiza una disquisición sobre los hidalgos, como uno de los defectos llevados a Portugal por los españoles. Ese afán de aparentar es exclusivamente español y para denostarlo echa mano de autoridades hispánicas, ya que en este fragmento cita a Cervantes y su crítica a la hidalguía pero, sorprendentemente, no se deberá a la parodia de los libros de caballerías, sino a las ínfulas de hidalga de Maritornes. En la misma línea, entonces, la crítica surge de versos de Góngora, de memoria, en un perfecto "portuñol":

Noutras partes o povo manifestou por forma idêntica o horror de ser espanhol, mas houve de aquietar-se: faltava-Ihe um chefe de cabeça que o guiasse e o ouro de Castela corria célere e, como dizia D. Luís de Gôngora:

Tudo se vende esto dia,

Tudo el dinero lo iguala 29

Resulta paradójico, a la vez que provocador que, para ejemplificar el desprecio hacia el portugués que se vende al español por un título nobiliario, por salvar la vida, por cobardía o por tantos otros motivos deleznables, se ilustre con versos de un español.

Por su parte, Quevedo descuella en tres ocasiones. Todas ellas resultan de filiación confusa, parecen más textos populares que él atribuye al escritor español por lecturas a través de otros. El primer caso aparece en el tomo II y va antecedido por otros dos versos sin filiación alguna, a los que vamos a dedicar unas líneas. Podríamos atribuirlos a Quevedo, ya que los cita con él, o decidir que son anónimos. Lo sorprendente es que se pueden encontrar asignados a otros escritores españoles o a ninguno en concreto, pero siempre en trabajos de autores portugueses de otras épocas. Es decir, parecen ser unos versos famosos entre los intelectuales portugueses, aunque no se atribuyan a su autor original o se usen solo en la lengua vecina con un prurito de pedantería. Los versos tal y como el abad los presenta dicen así: "Politica, politica! a quanto obligas, haces que sean blancas las hormigas» 30 . Estos mismos versos los encontramos dos veces en Ensaio biographico-critico sobre os melhores poetas portuguezes ${ }^{31}$ atribuidos a Lope de Vega y reproducidos según esta variante: «Fuerza del consonante a quanto 
obligas! Haces que sean blancas las hormigas!»32, que trata sobre la necesidad de adaptar la lengua a la rima para poder cumplir las exigencias métricas. Por su parte, Alexandre Herculano usa el mismo dístico - no sabría decir si bien traído- para rebatir a un antagonista político con el cual porfía sobre el monumento a D. Pedro, duque de Bragança ${ }^{33}$. No solo se encuentra en obras portuguesas, ya que podemos ver varios textos españoles del siglo XIX y principios del XX, también sin referencia de autor. Son bastantes numerosos y baste aquí mencionar su procedencia española y latinoamericana, tanto de Brasil como de países de habla hispana. Solo voy a resaltar tres de entre todos ellos. Los más antiguos, por un lado, y aquellos que ofrecen los versos en el contexto de un pequeño proverbio narrativo, por otro. En boca del padre D. Simón López, en dos ocasiones, ya en el periódico de 1813 El procurador general de la nación y del rey, que emite un dictamen sobre la legalidad de la Inquisición, publicado de forma independiente ${ }^{34}$, ya en un volumen sobre la moral de las comedias ${ }^{35}$; en las Cartas críticas del padre Alvarado $36 ;$ y en varias obras misceláneas de los siglos XIX y XX, en español y en portugués podemos encontrar una estrofa de cuatro versos - populares, antiguos, que dijo el poeta o calificativos similares-, que reza así: «Muriose el cigarrón, tendió sus ancas, / y cargaron con él hormigas blancas: / fuerza del consonante, a lo que obligas, / que haces que sean blancas las hormigas».

Aparentemente, lo que encontramos en estos eruditos portugueses del siglo XIX forma parte de una costumbre lectora a través de otras voces y miradas, sobre todo de tipo político, pero también de sátira literaria, para demostrar erudición -rayana en la pedantería- perpetuando una cultura hispánica no siempre correcta en sus fuentes, tal y como se ve en los autores españoles, que citan los versos como sustrato común de los proverbios populares. Lo curioso es que las voces tienen vínculos con el lector, hermanados por la sotana, ya que López y Alvarado son eclesiásticos, además de que Alvarado residió en Tavira, Portugal, con la llegada de los portugueses a Sevilla. La premisa de la educación literaria de frontera se cumple con creces en este entramado de citas eruditas: se lee lo próximo en espacio e ideología, no lo que existe o no lo que se quiere.

Aparte de estos versos misteriosos en español, la cita que atribuye a Quevedo es «No olvides es comedia nuestra vida y teatro de farsa el mundo todo» ${ }^{37}$. Por muy conocidos resulta todavía más curioso que ponga en boca de Quevedo versos de Calderón de la Barca. Y todavía más si lo repite de nuevo en el tomo X, a propósito de un homenaje que la ciudad le rinde en 1935. Sin embargo, la guinda del pastel de este enredo de lecturas españolas, con o sin fundamento libresco, se encuentra en

32. Silva, 1851 , pp. 130 y 178.

33. Herculano 1873-1908. Acceso restringido en <http://catalogo.bnportugal.gov.pt/ipac20/ipac.jsp?p rofile=bn\&source= $\sim$ !bnp\&view=subscriptionsummary\&uri=full=3100024 !873916 ! $2 \&$ ri $=1$ \&aspect=su btab13\&menu=search\&ipp=20\&spp=20\&staffonly=\&term=lus\%C3\%83\%C2\%ADadas\&index=. TW\&uinde $\mathrm{x}=$ \&aspect $=$ subtab13\&menu=search\&ri $=1>$

34. López, 1813, p. 9.

35. López, 1814, p. 209

36. Alvarado, 1825, p. 231

37. Alves, 2000 , tomo II, p. 355

HIPOGRIFO, 7.1, 2019 (pp. 597-613) 
un volumen de 1729, segundo tomo de las obras completas de Quevedo, guardado en el ADB, donde aparece en nota manuscrita al final del volumen «no olvides, es comedia nuestra vida». Parece obvio que el abad leyó esta nota, que no comprobó su procedencia, y también resulta evidente que, aunque no tenga en su biblioteca personal obras de Quevedo, tenía acceso a las de otros hombres doctos de la ciudad, del Seminario, la Junta y la Mitra.

Por último, en el mismo tomo VIII de sabor español, surge el Quevedo más irónico, con los versos siguientes: «Monjas claras / claro está / no tien razón / dar este chocolate, / sin decir: agua va» ${ }^{38}$. De manera amable, Alves recuerda que existen usos y costumbres similares a un lado y otro de la raya, al enumerar algunas normas municipales de finales del siglo XVII, entre ella la de avisar del desagüe manual, so pena de multa. Buscados los versos en la obra de Quevedo, aparecen mencionados a través de otros eruditos, en lo que parece una lectura a través de otros, una cita indirecta. Así, un contemporáneo al abad y cercano en el espacio transfronterizo, Juan Domínguez Berrueta, publica una obra laudatoria sobre Salamanca ${ }^{39}$, donde aparece la alusión dicha anécdota del escritor barroco, que parece tener mucho de leyenda:

Nos contaba nuestra abuela, cuando éramos chicos, aquella leyenda del chocolate de Quevedo. Convidaron las monjas Claras al poeta a tomar chocolate, y para oír sus ocurrencias, se lo presentaron excesivamente acuoso el ponderado soconusco monjil. Quevedo tomó lo que le dieron y obsequió a las bromistas monjas con esta copla: Monjas Claras, claro está, / pero es grande disparate / dar agua por chocolate, / y no decir: jagua va! ${ }^{40}$

\section{CONCLUSIONES}

No se puede sino reflexionar sobre esta cultura española, sobre esta educación literaria hispánica del Siglo de Oro en Portugal desde el enfoque de la ciudad como entidad histórica, desde los fondos bibliográficos españoles en la frontera hispanolusa y desde el ejemplo del lector transfronterizo erudito del Siglo de Oro.

La lucha de Bragança a lo largo de los siglos no se ha centrado únicamente en la línea de la frontera física y bélica, sino también en la creación de una opinión pública favorable a su territorio, ya sea frío, ya se encuentre interior, ya esté despoblado, ya continúe ruralizado: el objetivo de la ciudad desde múltiples entidades es mostrar todo su atractivo y esplendor. Al mismo tiempo, el bragançano no se suma completamente al proyecto transformador cultural que aúne patrimonio no folclórico, alta cultura y turismo patrimonial de cariz intelectual, probablemente por desconocimiento.

El análisis de este legado del ADB, tanto en su existencia y conservación como en su contenido, ofrece una radiografía de la educación literaria y cultural extranjera

38. Alves, 2000, tomo VIII, p. 188

39. Domínguez Berrueta, 1938

40. Domínguez Berrueta, 1938, p. 84

HIPOGRIFO, 7.1, 2019 (pp. 597-613) 
a lo largo de los siglos de una sociedad transfronteriza y refrenda las hipótesis aquí expuestas, además de brindar algunos ejemplares prometedores que puedan ser más valiosos en su contenido histórico, literario o cultural, por el hecho de poseer menos ejemplares o ser menos accesibles.

Por último, Alves no domina las lecturas de Quevedo o Góngora, teniendo en cuenta que confunde citas, las repite, aunque sean erróneas, además de que las apariciones escritas son escasas. De hecho, el acercamiento del abad a la cultura española manifiesta dos realidades. Por un lado, que es perfectamente coherente con sus lecturas y sus escritos. En su biblioteca personal se conserva su Quijote, cuya lectura atenta lo ha convertido en un experto desconocido de la obra cervantina, mientras que no existe ninguna obra de Quevedo, Góngora o Lope y por ello no domina sus obras, citando erróneamente o a través de otros. Por otro lado, explicita perfectamente que las referencias al país vecino como un plus de calidad, como una autoridad, como una fuerza cultural añadida a las argumentaciones etnográficas portuguesas son constantes y necesarias para justificar el rumbo de sus estudios del distrito de Bragança, frontera sin fronteras.

Los términos rayanos que más se usan en zona transmontana desde el punto de vista del capital simbólico son territorio, identidad y cultura, con la tendencia de hacerse diferentes recuperando y conservando antiguas formas de vida, más sostenibles, para encarar el futuro de una forma responsable y respetuosa, pero sin dejar de vivir la frontera como una manera más de ser Otro portugués. Esa identidad territorial y cultural que ofrece momentos deliciosos como estos, de prácticas cotidianas extraordinarias, recogidas, una vez más, por el abad:

Estas tres povoaçoens de Santhiago, Rubiaes e Meaos são limitrophas de ambos os Reinos de Portugal e Hespanha... Nestas povoações ambas as coroas appresentão Justiças Criminaes, e quanto ao Civel os mesmos Povos são os que elegem Juizes, do quem as Partes appellão ou para o Ouvidor de Bragança, se são portuguezes, ou para algum Corregedor de Hespanha, sendo hespanhoes.

Quando cazão, têm a liberdade de se constituhirem hespanhoes ou portuguezes, bebendo hum copo de vinho á saude do monarca de quem querem ser vassalos, e depois com esta mesma cerimonia vão erigir dois lares na caza que handem habitar, para assim escaparem aos procedimentos que de qualquer dos reinos se queira ter contra elles, fugindo ora para este ora para aquelle lar opposto ás Justiças que os buscão.

Dizem elles ter o previlegio concedido por ambas as coroas de conduzirem livremente, sem pagar cousa alguma de hum a outro reino, todos os generos, e fazendas de que precisarem; e que são izentos de servir, nem no militar nem no civil, a qualquer das duas coroas, a quem unicamente só reconhecem com o tributo annual de mil e duzentos reis, que a ambas paga cada individuo ${ }^{41}$. 


\section{BiBLIOgRAFÍA}

Alvarado, Francisco, Cartas críticas que escribió el Rmo. Padre Maestro Fr. Francisco Alvarado..., Madrid, E. Aguado, 1825.

Alves, Francisco Manuel, Memórias arqueológico-históricas do distrito de Bragança, coord. Gaspar Martins Pereira, 2. ${ }^{a}$ ed., Bragança, Câmara Municipal de Bragança/Instituto Português de Museus, 2000, 12 tomos.

Cervantes Saavedra, Miguel de, Don Quijote de la Mancha, ed. Francisco Rico, Barcelona, Círculo de Lectores/Galaxia Gutenberg, 2004.

Domínguez Berrueta, Juan, Salamanca. Guía sentimental, 3. ${ }^{a}$ ed., Salamanca, Calatrava, 1938.

Dotras Bravo, Alexia, «La biblioteca cervantina de Carolina Michaëlis de Vasconcelos en la Universidade de Coimbra», en Rumbos del Hispanismo en el umbral del Cincuentenario de la AlH, vol. III, Siglo de Oro (Prosa y poesía), ed. Patrizia Botta, Roma, Bagatto Libri, 2012, pp. 167-173.

Dotras Bravo, Alexia, «Las falsificaciones de la historia: Cervantes entre Bragança y Sanabria», Boletín de la Biblioteca de Menéndez Pelayo, XCII, 2016a, pp. 115-131.

Dotras Bravo, Alexia, «Leer y representar clásicos siempre (re)escritos. Angélica y Medoro del barroco a la corte de Fernando VI», Atalanta, 4.2, 2016b, pp. 137154. Disponible en <http://www.revistaatalanta.com/index.php/ARLB/article/ view/10.14643-42E/71>.

Dotras Bravo, Alexia, «La recepción de Miguel de Cervantes en el Portugal contemporáneo», Edad de Oro, 35, 2016c, pp. 67-78.

Dotras Bravo, Alexia, «La recepción más desconocida de Cervantes en Portugal», en Cervantes y Portugal: Historia, Arte y Literatura/Cervantes e Portugal: História, Arte e Literatura, ed. Aurelio Vargas Díaz-Toledo y José Manuel Lucía Megías, Oporto, Estratégias Criativas/Fundação Calouste Gulbenkian, 2018, pp. 237-254.

Dotras Bravo, Alexia, «La presencia de la lengua y cultura españolas en los archivos del saber portugués en la época de la Contrarreforma: el Arquivo Distrital de Bragança», en Culturas, Identidades e Litero-Línguas Estrangeiras. Atas do II Colóquio Internacional de Línguas Estrangeiras, ed. Alexia Dotras Bravo et al., en prensa.

Fernandes, Armando, Constrastes e transformações na cidade de Bragança. 19742004, Bragança, Câmara Municipal de Bragança, 2004.

Herculano, Alexandre, Opúsculos VIII, Lisboa, Viúva Bertrand, 1873-1908.

Jacob, João, Cidades e vilas de Portugal. Bragança, Lisboa, Editorial Presença, 1997. 
López, Simón, Dictamen del señor D. Simón López, diputado en Cortes..., Valencia, El Milagro, 1813.

López, Simón, Pantoja o resolución histórica teológica de un caso práctico de moral sobre comedias, Murcia, Herederos de Muñiz, 1814.

Nunes, António Jorge (coord.), Comemoração dos 545 anos de Bragança cidade, Bragança, Câmara Municipal de Bragança, 2009.

Pereira, Vítor, «Humanização, audácia e inovação colocaram Bragança no topo das Smart cities», Bragança. Suplemento de Villas\&Golfe, 90, novembro 2016, p. 96.

Ribeiro da Silva, Francisco, «Guerras de 1640. Guerra da sucessão de Espanha. Guerra dos Sete Anos», en Francisco Manuel Alves, Memórias arqueológico-históricas do distrito de Bragança, coord. Gaspar Martins Pereira, Bragança, Câmara Municipal de Bragança/Instituto Português de Museus,vol I, pp. 171-174.

Rodrigues Monteiro, José, 800 anos de Bragança no $8^{\circ}$ centenário do $7^{\circ}$ foral, Bragança, Câmara Municipal de Bragança/Instituto Politécnico de Bragança, 1988.

Silva, José Maria da Costa e, Ensaio biographico-critico sobre os melhores poetas portuguezes, Lisboa, Imprensa Silviana, 1851.

Sousa, Fernando de, História da Indústria das Sedas em Trás-os-Montes, vol. 1, Porto, edições Afrontamento, 2006.

Sousa, Fernando de (coord.), Bragança na época contemporânea (1820-2012), Bragança, Câmara Municipal de Bragança, 2013. 